\title{
Capacitive Sensor Mats for Pressure Detection with High Sensitivity
}

\author{
Holger Böse, Eric Fuß, Johannes Ehrlich \\ Fraunhofer-Institut für Silicatforschung, Neunerplatz 2, 97082 Würzburg, Germany, \\ E-mail: holger.boese@isc.fraunhofer.de
}

\begin{abstract}
New flexible pressure sensor mats based on dielectric elastomers are described in this paper. They can be applied for the detection of compression loads on rigid as well as on compliant substrates. The measurements with the sensor mats exploit a capacitive principle, where the electric capacitance between integrated flexible electrodes is changed upon compression. The basic design of the sensor mats comprises two flexible profiles which include a thin elastomer film in a sandwich configuration. When the sensor mat is compressed, the profiles penetrate into each other and stretch the elastomer film, causing an increase of the capacitance between the electrode layers. The sensitivity and other characteristics of the sensor mats are determined by the shape of the profiles, the location of the electrode layers and properties of the elastomer materials used and can be tuned by these parameters. Some examples of such compression sensor mats are introduced and discussed. Intended applications are seat occupation surveillance, load detection of the human body or impact detection of machines and robots.
\end{abstract}

Key words: Pressure sensors, compression load, flexible sensor mat, compliant substrate, silicone elastomer.

\section{Introduction}

Mechanical sensors for the detection of force, load or pressure are required in many technical applications in various branches such as automotive, machine tools, robotics, sports and leisure equipment or medical technology. Today, mostly strain gauges are used for these purposes, where the change of electric resistance upon variable loads on the sensor is measured. Strain gauges are not very flexible sensors and can primarily be integrated in rigid mechanical structures.

However, load measurements in soft or compliant environments such as on seat surfaces or on the human body also need suitable equipment to be conducted. This task may even include the detection of loads on extended surfaces like beds in order to prevent bedsores on the skin of immobile patients. For this purpose, flexible sensor mats which exhibit high sensitivity are required.

Some flexible sensor mats are already commercially available, but they are quite sophisticated, very expensive and therefore restricted to niche applications. In order to fill this gap, simple and sensitive flexible sensor mats based on dielectric elastomers are introduced in this paper. Dielectric elastomers (DE) are basically thin elastomer films (thickness about $100 \mu \mathrm{m}$ ) which are coated with stretchable electrode layers on both sides [1]. When the DE film is stretched in one or two dimensions, the electrical capacitance between the electrodes is enhanced due to the increase of electrode surface and the simultaneous decrease of the film thickness (Fig. 1). By this way, a very effective and simple stretch sensor is achieved which can be used in applications in which stretch detection is required. Some work on the properties of DE sensors and their applications has been published [2-6]. A special use is the integration of DE sensors in corresponding dielectric elastomer actuators to monitor their actuation state [7-9]

However, the simple DE film sensors are very insensitive for the detection of pressure loads acting on the film surface. Therefore, new concepts for pressure-sensitive sensor mats based on flexible profiled structures have been developed [10,11]. The objectives of this paper are to outline the basic working principle of the sensor mats, to describe their geometrical design and their properties and to demonstrate the wide variability of their characteristics in terms of capacitance vs. compression load. 


\section{Sensor Principle}

State-of-the-art DE films are highly stretchable and correspondingly exhibit large increases of capacitance when stretched. This is schematically shown in Fig. 1. In contrast, when the DE film is compressed between rigid surfaces, no significant enhancement of capacitance occurs at least at moderate pressures of some bar due to the volume incompressibility of the elastomer (Fig. 2).

In order to enhance the sensitivity for compression loads drastically, new types of sensor mats have been introduced recently [10]. The basic principle of the sensor mat is shown in Fig. 3. The DE elastomer film is embedded between two profiled surfaces in a sandwich configuration. When the sensor mat is compressed, the profiles penetrate into each other, stretching the DE film. This conversion of pressure load to strain leads to a large increase of the capacitance of the DE film. The profiles and the DE film both consist of silicone elastomers.

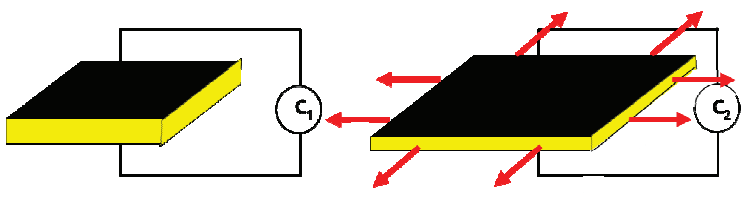

Fig. 1. Increase of capacitance upon stretching dielectric elastomer film.
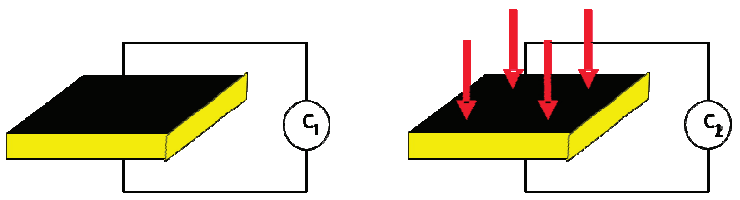

Fig. 2. No increase of capacitance by compression load on the dielectric elastomer film.

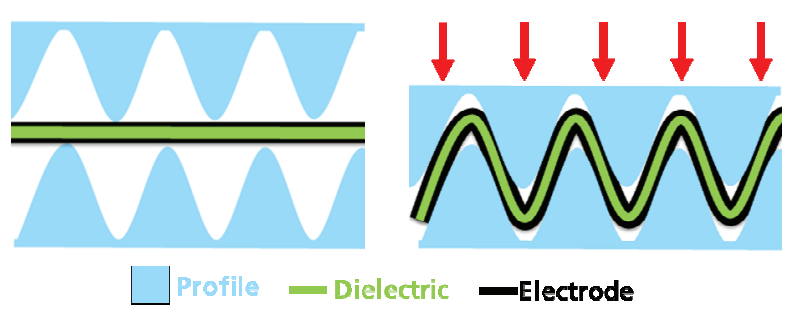

Fig. 3. Scheme of new sensor mat with wave profiles.

\section{Sensor Mat Design and Properties}

A sensor mat derived from the basic principle shown in Fig. 3 was manufactured and investigated. In order to shield the capacitor from external electric fields, the DE film contains three electrode layers where the two outer layers are set to ground potential. The capacitance is measured between the inner and outer electrode layers, thus doubling the capacitance compared with the configuration in Fig. 3. For a DE film with an electrode area of about $100 \mathrm{~mm} \times 100 \mathrm{~mm}$ and a thickness of the dielectric between the electrode layers of 80 $\mu \mathrm{m}$, the capacitance amounts to approx. $6 \mathrm{nF}$. Fig. 4 depicts the characteristics of the sensor mat with the DE film between two wave-shaped profiles upon a stepwise enhancement of the compression load. Up to a load of $1000 \mathrm{~N}$, the capacitance is nearly doubled. For comparison, the corresponding behavior of a pure DE sensor film compressed between rigid surfaces in the same way is also shown in Fig. 4. It is clearly visible that no detectable capacitance change occurs with the pure DE sensor film, demonstrating the striking advantage of the new sensor principle. Fig. 5 depicts a photograph of the sensor mat with the wave profiles.

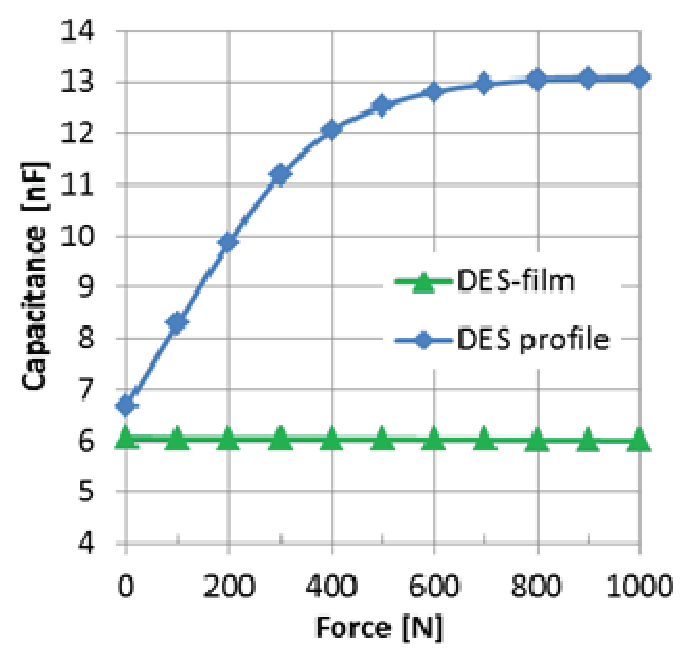

Fig. 4. Dependence of capacitance of new sensor mat with wave profiles on load force compared with pure dielectric elastomer film.

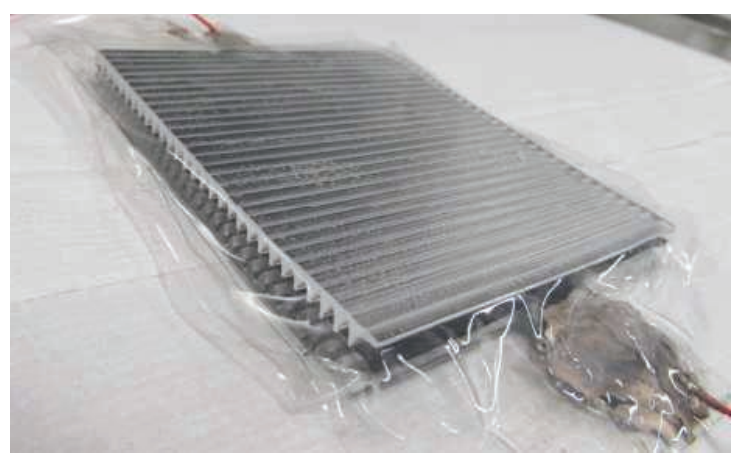

Fig. 5. Sensor mat with wave profiles (area about $100 \mathrm{~mm} \times 100 \mathrm{~mm}$, thickness $8 \mathrm{~mm}$ ). 
A modified configuration of the compression sensor mat with wave profiles is shown in the scheme in Fig. 6. It differs from the former sensor mat by the location of the electrode layers. Here, the outer electrodes are shifted from the DE film to the wave profile surfaces. Only the inner electrode layer remains in the elastomer film. A sensor mat of this type is shown in Fig. 7.

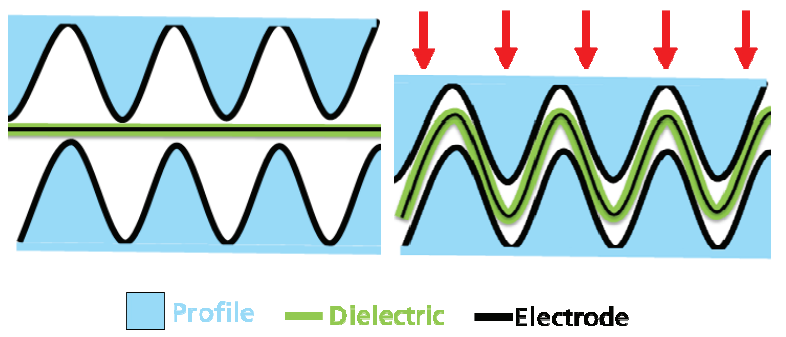

Fig. 6. Scheme of the sensor mat with wave profiles coated with electrode layers.

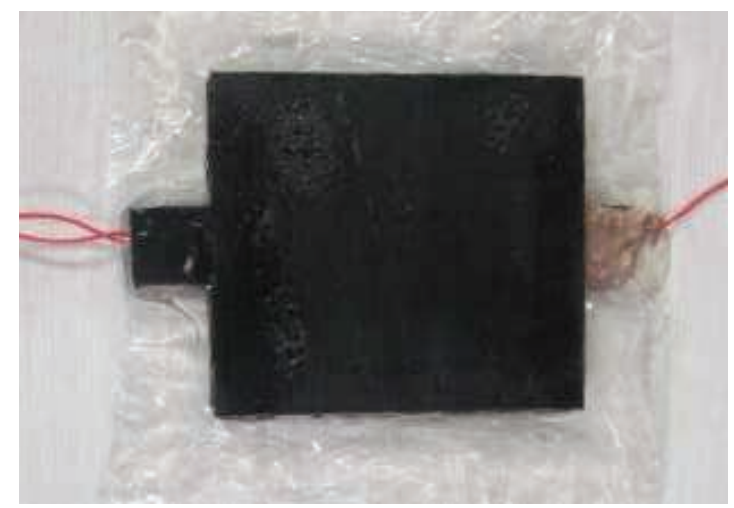

Fig. 7. Sensor mat with wave profiles coated with electrode layers (area about $100 \mathrm{~mm} \times 100 \mathrm{~mm}$, thickness $8 \mathrm{~mm}$ ).

The displacement of the outer electrode layers to the profiles has a strong impact on the characteristics of the sensor mat. Due to the considerably larger distance of the inner and outer electrodes, the initial capacitance in the uncompressed state of the sensor mat is much smaller. The dependence of the capacitance of the sensor mat with electrode layers on the wave profiles compared with the corresponding sensor mat without electrode layers on the profiles is depicted in Fig. 8. The capacitance starts from about $0.6 \mathrm{nF}$ and rises up to $12 \mathrm{nF}$ at a load of $1000 \mathrm{~N}$, which gives an increase factor of 20, surmounting the corresponding factor of the first sensor mat strongly.

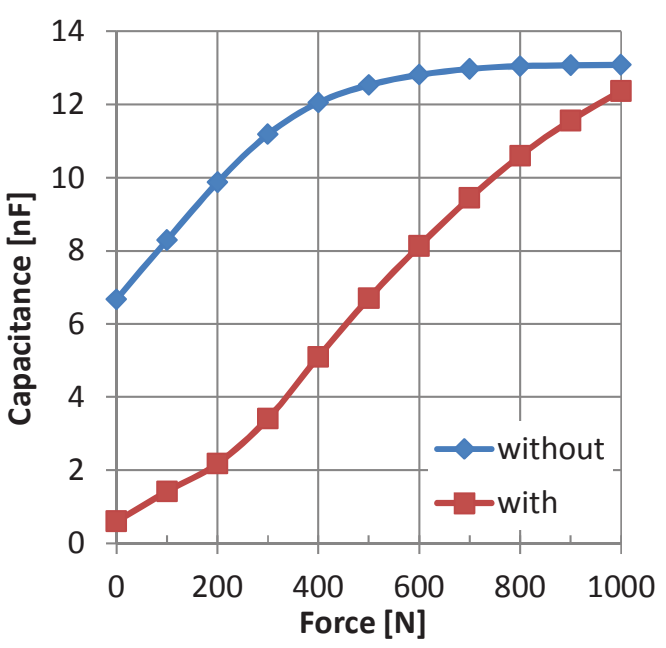

Fig. 8. Dependence of capacitance of sensor mats with wave profiles without / with electrode layers on load force.

The wave contour of the profiles is only one possible shape. Another profile shape is schematically shown in Fig. 9. Here, the waves which extend in one dimension are replaced by a quadratically arranged pattern of small knots. As before for the wave shape, in a first design the DE film in the center contains three electrode layers and the profiles carry no electrode layers. Another configuration of the sensor mat with knot profiles is schematically depicted in Fig. 10. As in case of the wave profiles, the profiles are now coated with electrode layers and the DE film contains only the inner electrode.

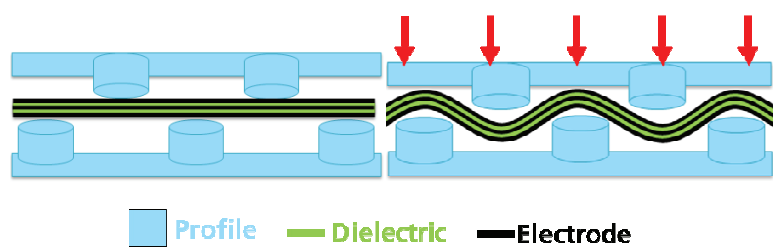

Fig. 9. Scheme of the sensor mat with knot profiles containing a dielectric elastomer film with 3 electrode layers.

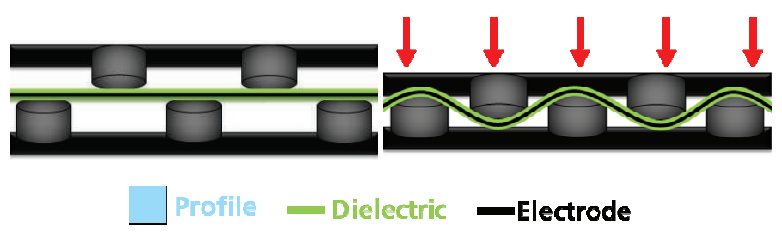

Fig. 10. Scheme of the sensor mat with knot profiles coated with electrode layers containing a dielectric elastomer film with 1 electrode layer. 
A photograph of the uncoated elastomer sheet with knot profile is shown in Fig. 11. Fig. 12 depicts a complete sensor mat according to the second type, where the knot profiles are coated with electrode layers. The electrode layers have a surface of about $100 \mathrm{~mm} \times 100 \mathrm{~mm}$ as in case of the sensor mats with wave profiles, but the sensor mats with knot profiles are considerably thinner ( $3 \mathrm{~mm}$ instead of $8 \mathrm{~mm}$ ). Furthermore, due to the two-dimensional arrangement of the knots on the profiles, the flexibility is the same in both dimensions, in contrast to the sensor mats with wave profiles.

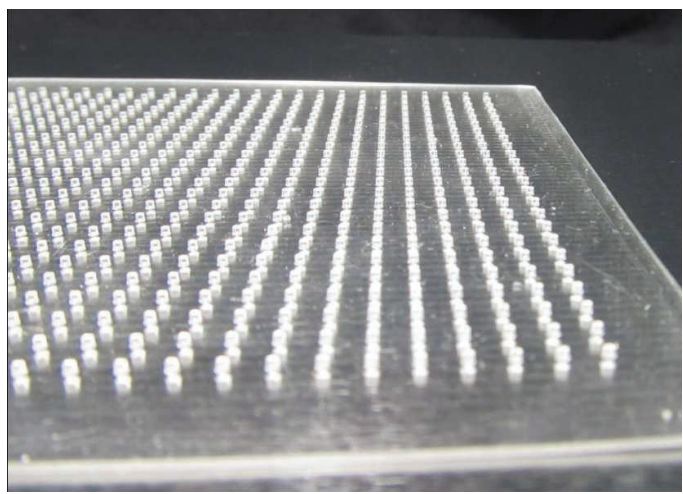

Fig. 11. Photograph of knot profile.

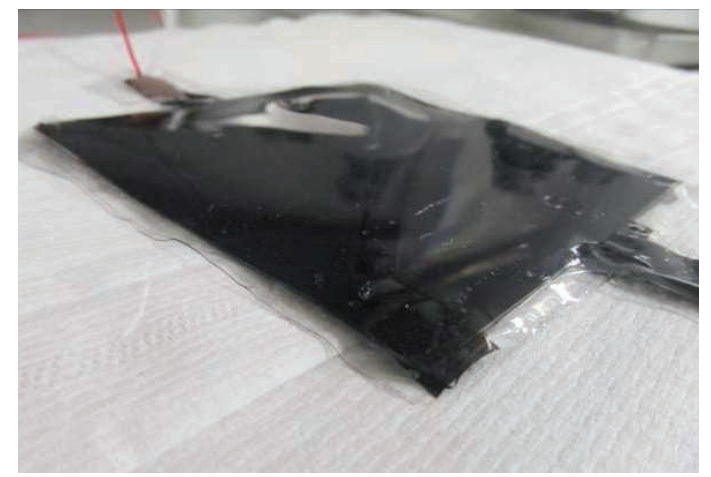

Fig. 12. Sensor mat with knot profiles (area about $100 \mathrm{~mm} \times 100 \mathrm{~mm}$, thickness $3 \mathrm{~mm}$ ).

In Fig. 13, the characteristics of the two sensor mats with knot profiles are compared with each other. The sensor mat without electrode layers on the profiles exhibits no significant change of the capacitance upon compression. The reason for this behavior is that almost no stretch of the DE film occurs between the knot profiles. In contrast, the sensor mat with electrode layers on the knot profiles exhibits a remarkable enhancement of the capacitance upon compression load. This large increase is attributed to the approach of the outer electrode layers on the profiles to the inner electrode. The increase factor of the capacitance at a load of
$1000 \mathrm{~N}$ is not as large as for the corresponding sensor mat with wave profiles, but with a figure of more than 10 it should be sufficient for most applications. In addition, with the considerably lower thickness and the higher flexibility, the sensor mat with coated knot profiles exhibits an advantageous combination of properties for compression load measurements on compliant surfaces.

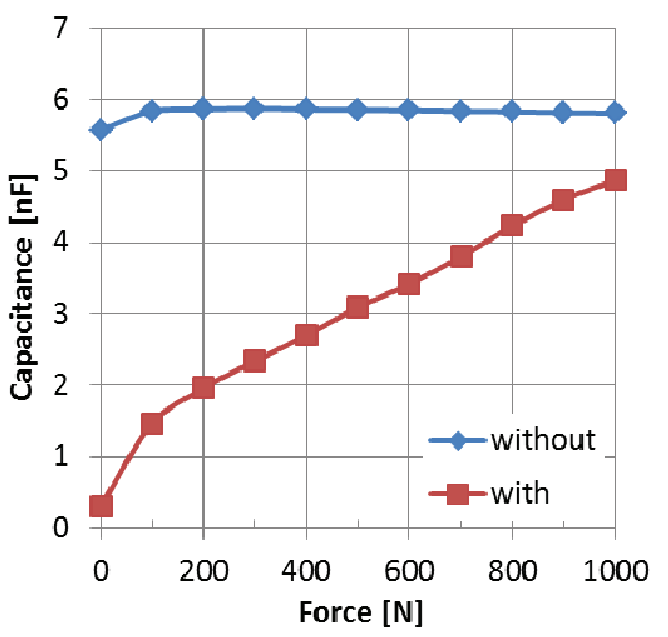

Fig. 13. Dependence of capacitance of sensor mats with knot profiles without / with electrode layers on load force.

The electrode layers in the sensor mats can also be split into separate segments. With this segmentation, the load distribution may be locally resolved. As an example, Fig. 14 depicts the dependence of capacitance of four separated electrode areas on a sensor mat with knot profiles on the applied compression load. Since the load on all four electrode segments is the same, the curves are very similar.

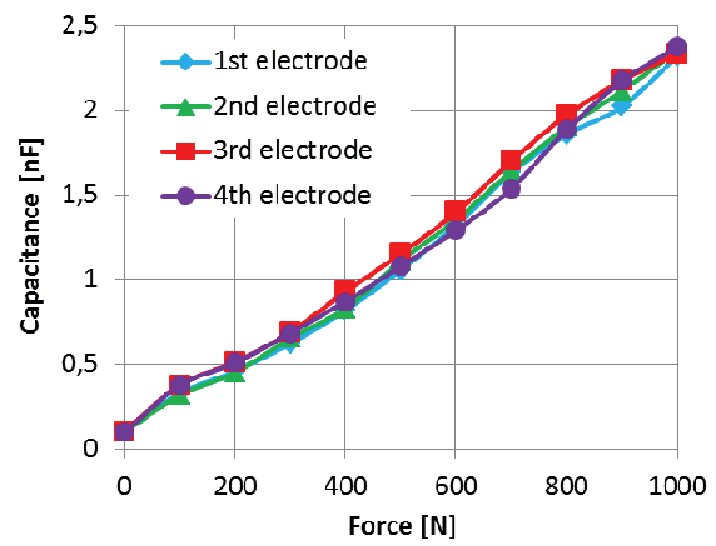

Fig. 14. Dependence of capacitance of sensor mat with knot profiles containing four separate electrode areas on load force. 


\section{Applications of the Sensor Mats}

A possible application of the compression sensor mats is the surveillance of seat occupation in vehicles. The sensor mat can not only detect the presence of a person, but it can also determine his or her weight in order to distinguish between heavy persons, mediumweight passengers and children. This signal may be used to control the airbag in case of an accident. Furthermore, if the load is measured locally resolved on different locations, the approximate position of the person can be determined as well. This information may be used for the airbag control. A demonstration setup for the detection of compression loads on a seat cushion is shown in Fig. 15. The cushion on the chair contains four sensor mats whose signals are displayed on the screen.

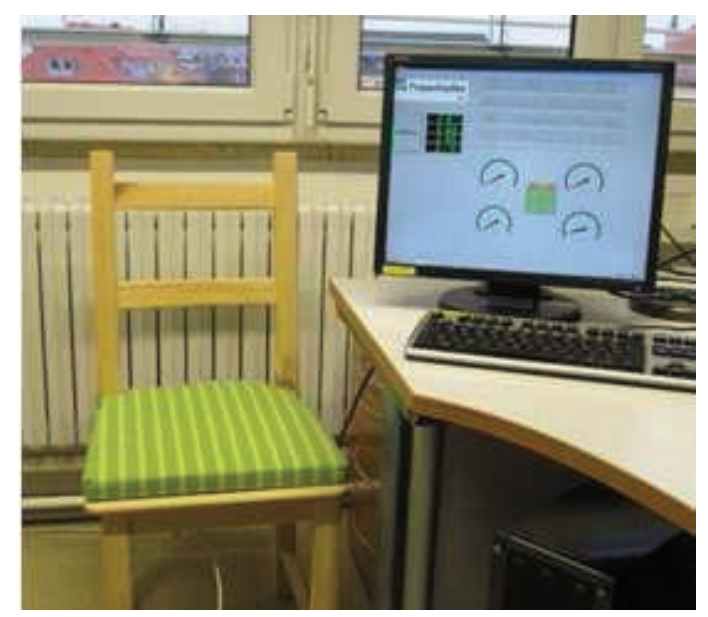

Fig. 15. Demonstration of the performance of a compression sensor mat for seat occupation surveillance.

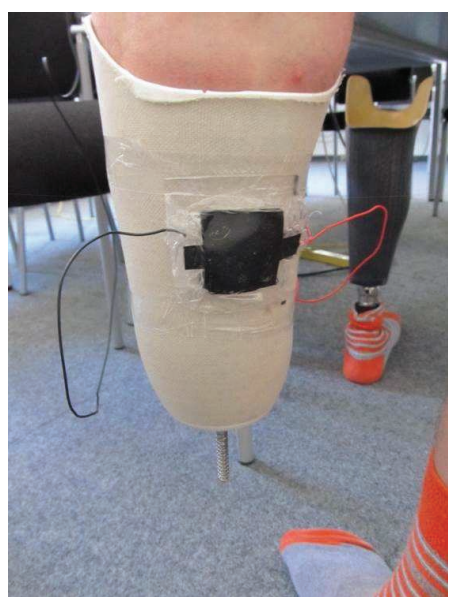

Fig. 16. Demonstration of the performance of a sensor mat for the detection of compression load in a leg prosthesis.
Another possible application of the sensor mats concerns the detection of pressure loads in a leg prosthesis. Too high local loads exerted by the prosthesis may cause sores on the skin of the amputee. This overload should be recorded before damages occur. Fig. 16 shows a first experiment in which the integration of a small compression sensor into a leg prosthesis was tested.

\section{Other Properties of DE Sensor Films}

For the use of dielectric elastomer sensors in practical applications, some other properties are also important. In order to evaluate such relevant properties, further investigations on pure dielectric elastomer sensor films were performed. In a first test, the long-term behavior of a DE film war studied. As shown in Fig. 17, the DE sensor film survived 400,000 cycles with a periodic stretch of $100 \%$ without significant change of the capacitance in the stretched and unstretched state.

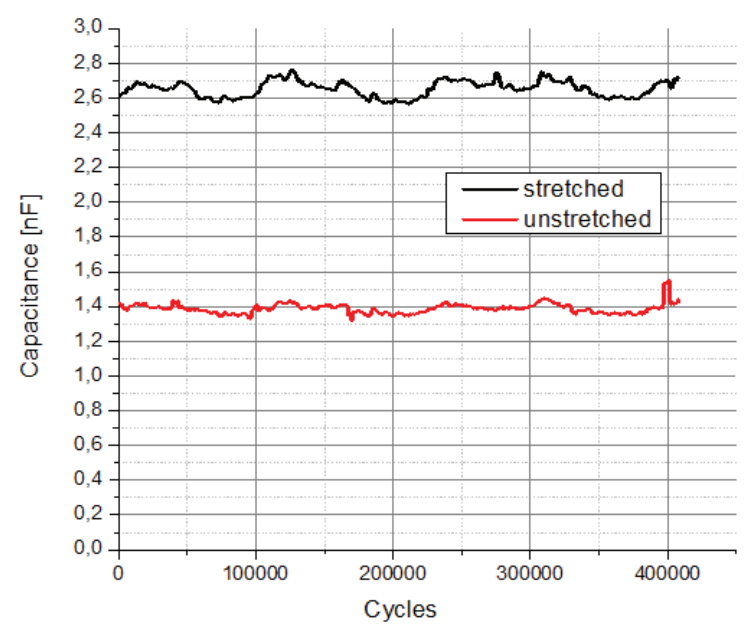

Fig. 17. Long-term behavior of a DE sensor film at cyclic stretch with $100 \%$ : Measured capacitance in the stretched and in the unstretched state over 400,000 cycles.

In another experiment, the temperature dependence of the capacitance of two DE sensor films without applied load was studied in an environmental chamber. Starting from ambient temperature, the temperature was lowered to $-30{ }^{\circ} \mathrm{C}$, then increased to $+80{ }^{\circ} \mathrm{C}$ and again lowered to the initial value. The total measured change in capacitance between the extreme temperatures was about $20 \%$, but no remaining change after the temperature cycle was observed. If required, the effect of temperature on the capacitance may be corrected by a reference measurement with an unloaded sensor element. 


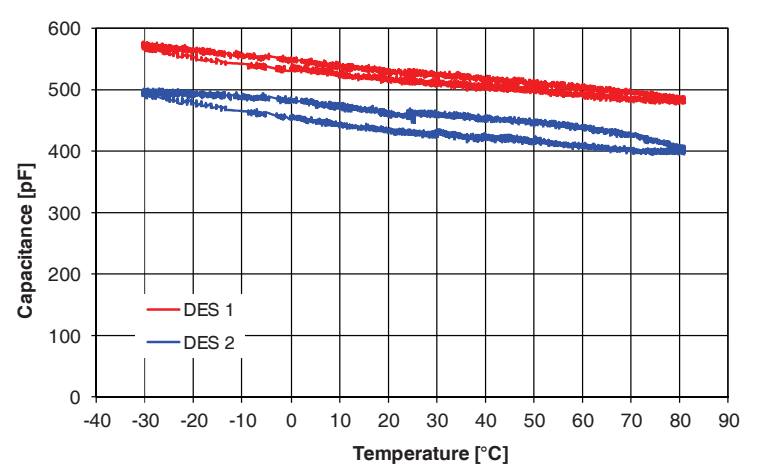

Fig. 18. Dependence of capacitance of two DE sensor films without load on temperature.

\section{Conclusion}

Various types of new sensor mats for the detection of compression loads on extended rigid as well as compliant surfaces were introduced. The sensor mats convert compression loads into tensile loads on an elastomer film which is located between two profiled elastomer components. A set of design parameters provides the possibility to influence the sensitivity and characteristics of the sensor mat in terms of the dependence of capacitance on compression load. The shape of the elastomer profiles determines the degree of strain of the elastomer film when the sensor mat is compressed. Profiles with wave and knot shape were introduced, where the wave profiles cause the largest stretch of the elastomer film. A strong impact is given by the locations of the electrode layers for the capacitance measurements. If the profile surfaces are coated with electrode layers, especially large factors of capacitance enhancement can be achieved.

The overall conclusion of the described investigations is that a number of design and material parameters exist which offer the opportunity to tune the sensor characteristics and match them to the requirements of a given application. There are many applications in which the detection of compression forces on extended rigid or flexible surfaces is required. The sensor mat can also be equipped with electrode patterns in order to measure the pressure distribution with local resolution. Examples for such applications are the seat occupation surveillance in vehicles or the monitoring of pressure distributions on the human body for medical purposes such as decubitus prophylaxis in beds and on wheelchairs or the detection of overloads in shoes or in a prosthesis. Further possible applications concern the collision detection of robots in human-machine interaction and the registration of walking on floors for safety reasons. The new simple and cost effective compression sensor technology offers farreaching perspectives to provide sensors whose characteristics are perfectly adapted to the given requirements.

\section{Acknowledgement}

Financial support for this work from the Bavarian State Ministry for Economy, Infrastructure, Traffic and Technology is gratefully acknowledged.

The authors thank Stephan Wirthmann, Philipp Lux, Pascal Engel, Konrad Beck and Valentin Oechsner for technical support.

\section{References}

[1] F. Carpi, D. De Rossi, R. Kornbluh, R. Pelrine, P.Sommer-Larsen : Dielectric Elastomers as Electromechanical Transducers, Elsevier, 2008

[2] M. Rosenthal, N. Bonwit, C. Duncheon, J. Heim: Applications of dielectric elastomer EPAM sensors, Proc. SPIE Vol. 6524 (2007) 65241F

[3] S. Son, N. C. Goulbourne: Finite deformations of tubular dielectric elastomer sensors. J. Intelligent Material Systems and Structures, Vol. 20 (2009) 2187-2199

[4] B. Kim, S. Shin, J. Chung, Y. Lee, J.D. Nam, H. Moon, H.R. Choi, and J.C. Koo: A dual axis shear force film sensor for robotic tactile applications, Proc. SPIE Vol. 7976 (2011) 28 1-5

[5] B. O’Brien, T. Gisby, I. A. Anderson: Stretch sensors for human body motion, Proc. SPIE Vol. 9056 (2014) 9056181 1-9

[6] D. Xu, T. G. McKay, S. Michel, I. A. Anderson: Enabling large scale capacitive sensing for dielectric elastomers, Proc. SPIE Vol. 9056 (2014) 90561A 1-18

[7] F.Carpi, G. Fantoni, D. De Rossi: Bubble-like dielectric elastomer actuator with integrated sensor: device and applications. Proc. Actuator (2006) 872-875

[8] K. Jung, K. J. Kim, H. R. Choi: A self-sensing dielectric elastomer actuator. Sensors and Actuators A: PhysicalVol. 143 (2008) 343-351

[9] N. H. Chuc, D. V. Thuy, J. Park, D. Kim, J. Koo, Y. Lee, J.-D. Nam, H. R. Choi: A dielectric elastomer actuator with self-sensing capability, Proc. SPIE Vol. 6927 (2008) 69270V

[10] H. Böse, E. Fuß: Novel dielectric elastomer sensors for compression load detection, Proc. SPIE Vol. 9056 (2014) 905614 1-13

[11] H. Böse, E. Fuß, P. Lux: Influence of design and material properties on the performance of dielectric elastomer compression sensors, SPIE Vol. 9430 (2015) 943079 1-12 